TRANSACTIONS OF THE

AMERICAN MATHEMATICAL SOCIETY

Volume 350, Number 10, October 1998, Pages 4235-4251

S 0002-9947(98)02026-1

\title{
UNIVERSAL MAPS ON TREES
}

\author{
CARL EBERHART AND J. B. FUGATE
}

\begin{abstract}
A map $f: R \rightarrow S$ of continua $R$ and $S$ is called a universal map from $R$ to $S$ if for any map $g: R \rightarrow S, f(x)=g(x)$ for some point $x \in R$. When $R$ and $S$ are trees, we characterize universal maps by reducing to the case of light minimal universal maps. The characterization uses the notions of combinatorial map and folded subedge of $R$.
\end{abstract}

\section{INTRODUCTION}

A map or mapping is a continuous function. A continuum is a compact, connected metric space. If $X$ and $Y$ are topological spaces, then a map $f: X \rightarrow Y$ is universal provided that if $g: X \rightarrow Y$ is a map, then there is a point $x \in X$ at which $f$ and $g$ agree, i.e., $f(x)=g(x)$. The notion is due to Holsztynski [2], who showed the following property.

Property 1.1. If $X$ is connected, $Y$ is an arc, and $f: X \rightarrow Y$ is a surjection, then $f$ is universal.

We prove this by establishing a related property.

Property 1.2. Suppose that $X$ is connected, and $Y$ contains an arc $a b$ whose complement is the union of two disjoint open sets $U$ and $V$ with $\bar{U} \backslash U \subset\{a\}$ and $\bar{V} \backslash V \subset\{b\}$. If $h, k: X \rightarrow Y$ are maps with $h(X) \subset a b \subset k(X)$, then $h$ and $k$ agree somewhere.

Proof. Assume that $h$ and $k$ do not agree anywhere. Order $a b$ so that $a<b$. Now let $L=k^{-1}(U) \cup\{x \in X \mid k(x) \in a b$ and $k(x)<h(x)\}$ and let $W=k^{-1}(V)$ $\cup\{x \in X \mid k(x) \in a b$ and $h(x)<k(x)\}$. Then $L$ and $W$ are disjoint nonempty open sets whose union is $X$. This contradicts the connectedness of $X$ and so $h$ and $k$ must agree somewhere.

Proof of 1.1. Let $g: X \rightarrow Y$ be a map. Apply 1.2 with $g=h$ and $f=k$.

Universal mappings are used in fixed point theory. A topological space has the fixed point property provided that every map $f: X \rightarrow X$ has a fixed point (i.e., $f(x)=x$ for some $x \in X$. Thus $X$ has the fixed point property if and only if the identity map on $X$ is universal. Moreover, if $f: X \rightarrow Y$ is universal, then $Y$ has the fixed point property (if $g: Y \rightarrow Y$ is a map, then $f$ and $g f$ must agree at some $x \in X$. Hence $f(x)$ is a fixed point of $g$.) Also, universal maps (to $T_{1}$ spaces) must

Received by the editors May 5, 1987 and, in revised form, January 21, 1997.

1991 Mathematics Subject Classification. Primary 54H25; Secondary 54F20.

Key words and phrases. Minimal universal, combinatorial, tree, fixed point property.

(C)1998 American Mathematical Society 
be surjections (otherwise, there would be a constant map $g$ which never agrees with f).

It follows quickly from 1.1 and this last remark that the universal maps to an arc are completely characterized as the surjective maps to the arc.

Holsztynski [3] characterized the universal maps to an $n$-ball as the AH-essential mappings (also see [4]). We wish to thank the referee for these references and also for their helpful location of errors in the first (and second) versions of this manuscript.

One can pose the general problem:

Problem 1.3. Suppose $X$ and $Y$ are continua and $Y$ has the fixed point property. Characterize the universal mappings from $X$ to $Y$.

A tree is a continuum which is the union of a finite collection of arcs, and contains no simple closed curve. It is well-known that trees have the fixed point property, and in fact universal mappings on trees have been studied by Marsh [5, 6]. In [5], he introduced the notion of a $\mathbf{u}$-mapping of trees and showed that all $u$-mappings are universal. The list of five properties which he used to define u-maps does not characterize universal maps on trees, and the goal of this paper is to completely characterize universal maps on trees (see Theorem 6.3).

We now establish some terms and notation. Let $R$ be a tree. Then between any pair of points $x$ and $y$ in $R$ there is a unique arc which we denote by $x y$. The order of a point $p \in R$, denoted by $\operatorname{ord}_{R}(p)$, is the number (necessarily finite) of components of the complement of the point. A point $p \in R$ is an endpoint of $R$ or branchpoint of $R$ according to whether $\operatorname{ord}_{R}(p)=1$ or $\operatorname{ord}_{R}(p) \geq 3$. A point of $R$ is a vertex of $R$ if it is either an endpoint or a branchpoint of $R$. We use the notation $E_{R}, B_{R}$, and $V_{R}$ to denote the set of endpoints, branchpoints, and vertices of $R$ respectively. If $x, y \in V_{R}$, then $x y$ is called an edge of $R$ provided $x y$ contains no vertex other than $x$ and $y$. If also $x$ or $y$ is an endpoint, then we call $x y$ a terminal edge of $R$.

Definition 1.4. If $x$ and $y$ are distinct points of $R$ with $x \notin E_{R}$, then by $C_{x}(y)$ we mean the closure of the component of $R \backslash\{x\}$ which contains $y$; clearly, $C_{x}(y)$ is a subtree of $R$ with a one-point boundary in $R$. More generally, a branch of $R$ is any subtree of $R$ with a one point boundary in $R$.

\section{A Reduction of the problem to the light CASE}

In this section, we will show that we can assume that we are working with a light minimal universal map.

The following lemma will be used several times in the paper. First, we introduce some convenient terminology.

Definition 2.1. Let $f: R \rightarrow S$ be a map of trees. Then a map $g: R \rightarrow S$ is said to miss $f$ provided that for all $x \in R, f(x) \neq g(x)$. Thus $f$ is universal if and only if no map from $R$ into $S$ misses $f$.

Lemma 2.2. Suppose that $f: R \rightarrow S$ is a map of trees which is not universal and $F=\left\{x_{1}, \cdots, x_{n}\right\}$ is a finite subset of $R$. Then there is a map $g: R \rightarrow S$ such that $g$ misses $f$ and $g(F) \subset E_{S}$.

Proof. Let $g^{\prime}: R \rightarrow S$ be a map which misses $f$. For each $i=1, \cdots, n$, let $K_{i}$ be a closed connected set containing $x_{i}$ in its interior such that $f\left(K_{i}\right)$ and $g^{\prime}\left(K_{i}\right)$ are 
disjoint. Since $F$ is finite, we can also choose the sets $K_{i}$ so that they are pairwise disjoint. Let $e_{i}$ be an endpoint of $S$ such that the arc from $e_{i}$ to $g^{\prime}\left(K_{i}\right)$ is disjoint from $f\left(K_{i}\right)$. Then $e_{i} g^{\prime}\left(x_{i}\right) \cup g^{\prime}\left(K_{i}\right)$ is a tree and hence an absolute retract; so we can define a map $h_{i}: K_{i} \rightarrow e_{i} g^{\prime}\left(x_{i}\right) \cup g^{\prime}\left(K_{i}\right)$ so that $h_{i}(t)=g^{\prime}(t)$ for $t$ in the boundary of $K_{i}$ and $h_{i}\left(x_{i}\right)=e_{i}$. Then the map $g: R \rightarrow S$ given by $g(x)=h_{i}(x)$ if $x$ lies in the interior of $K_{i}$ and $g(x)=g^{\prime}(x)$ otherwise is the desired map.

In what follows, we will say that the map $g$ constructed as in 2.2 is endpoint valued on $F$.

A map is light if the point inverses are totally disconnected and monotone if the point inverses are connected. Let $f: X \rightarrow Y$ be a map of continua. Let $M$ denote the decomposition space whose points are the components of point inverses of $f$, and let $m$ denote the natural mapping from $X$ onto $M$. Then $m$ is monotone and the induced map $l: M \rightarrow Y$ is light. This factorization is called the monotone-light factorization of $f$. It is shown in [8][page 165] that if $X$ is a tree, then so is $M$. We shall refer to $M$ as the middle space of $f$. If $g: X \rightarrow Y$ is a map which is constant on the components of point inverses under $f$, then there is a unique map $\hat{g}: M \rightarrow Y$ such that $g=\hat{g} m$. We shall describe this by saying $g$ factors through the middle space of $f$.

Lemma 2.3. Suppose $f, g:[0,1] \rightarrow S$ are maps which miss each other, with $f$ non-constant. Then there is a map $h:[0,1] \rightarrow S$ which misses $f$, agrees with $g$ at 0 and 1 , and factors through the middle space of $f$.

Proof. Assume for the moment that $g([0,1])$ and $f([0,1])$ are disjoint. If $g(0)=$ $g(1)$, let $h([0,1])=g(0)$. Otherwise, let $A$ be an arc in $g([0,1])$ from $g(0)$ to $g(1)$, and let $I$ be the middle space of the map $f$. Since $f$ is nonconstant, $I$ is an arc. Let $\eta:[0,1] \rightarrow I$ be the natural map and let $p: I \rightarrow A$ be a homeomorphism with $p(\eta(0))=g(0)$ and $p(\eta(1))=g(1)$. Then $h=p \eta$ is the required map.

Now in case $g([0,1])$ and $f([0,1])$ overlap, we can partition $[0,1]$ into finitely many subintervals $[a, b]$ such that $f([a, b])$ and $g([a, b])$ are disjoint for each subinterval $[a, b]$. Apply the above construction to each subinterval and let $h$ be the join of the maps obtained.

Theorem 2.4. Suppose that $f: R \rightarrow S$ is a map of trees which is not universal. Then there is a map $g: R \rightarrow S$ which misses $f$ and factors through the middle space of $f$.

Proof. By 2.2, there is a map $g^{\prime}: R \rightarrow S$ such that $g^{\prime}$ misses $f$ and $g^{\prime}\left(V_{R}\right) \subset E_{S}$. There are only finitely many components $C_{i}$ of point inverses of $f$ which contain a vertex of $R$. For each such $C_{i}$, choose a subtree $T_{i}$ of $R$ containing $C_{i}$ in its interior such that the boundary points of $T_{i}$ in $R$ are components of point inverses of $f$ and $f\left(T_{i}\right)$ is disjoint from $g^{\prime}\left(T_{i}\right)$. We can do this because nondegenerate connected subsets of trees have nonempty interior and so there are only countably many nondegenerate components of point inverses of $f$. Further choose the $T_{i}$ 's so that distinct $T_{i}$ 's are disjoint.

For each $i$, modify $g^{\prime}$ on $T_{i}$ as follows: First note that $f\left(T_{i}\right)$ and $g^{\prime}\left(T_{i}\right)$ are disjoint trees in $S$, and $g^{\prime}\left(T_{i}\right)$ contains an endpoint $e$ of $S$. Since $g^{\prime}\left(T_{i}\right)$ is an absolute retract, there is a map $g_{i}: T_{i} \rightarrow g^{\prime}\left(T_{i}\right)$ such that $g_{i}(t)=g^{\prime}(t)$ for $t$ in the boundary of $T_{i}$ and $g_{i}\left(C_{i}\right)=\{e\}$. Then the map $l$ which is $g^{\prime}$ off of the union of the $T_{i}$ 's and, for each $i$, is $g_{i}$ on $T_{i}$, misses $f$ and is constant on each component of a point inverse of $f$ which contains a vertex. 
We now modify $l$, using 2.3. Note that any component of a point inverse of $f$ on which $l$ is not constant is contained in the interior of an edge of $R$. For each such edge $p_{i} q_{i}$ of $R$, let $a_{i} b_{i}$ be the arc such that $p_{i} a_{i}$ and $b_{i} q_{i}$ are components in $p_{i} q_{i}$ of $f^{-1}\left(f\left(p_{i}\right)\right)$ and $f^{-1}\left(f\left(q_{i}\right)\right)$ respectively. Apply 2.3 to each $a_{i} b_{i}$ to obtain maps $h_{i}: a_{i} b_{i} \rightarrow S$ which miss $f$ on $a_{i} b_{i}$, agree with $l$ at $a_{i}$ and $b_{i}$, and are constant on the components of point inverses of $f$ lying in $a_{i} b_{i}$. The map $g$ which is $l$ off of the union of the $a_{i} b_{i}$ and, for each $i$, is $h_{i}$ on $a_{i} b_{i}$ is the desired mapping.

Theorem 2.5. A map of trees is universal if and only if the light factor of its monotone-light factorization is universal.

Proof. Let $f: R \rightarrow S$ be a map of trees and $f=l m$ be the monotone light factorization of $f$. It is readily checked that if the composition of two maps is universal, then the second map is also; so $l$ must be universal if $f$ is. For the converse, note first that since $l$ is light and $m$ is monotone, the components of the point inverses of $f$ are precisely the point inverses of $m$. Suppose that $f$ is not universal and let $g$ be a map missing $f$ which satisfies Theorem 2.4. Then the induced map $\hat{g}$ defined on the domain of $l$ by $\hat{g}(x)=g\left(m^{-1}(x)\right)$ misses $l$. Hence we conclude that $l m$ is universal if $l$ is universal.

Suppose that $f: X \rightarrow Y$ is a mapping, $A \subset X$ and let $f^{\prime}$ denote $\left.f\right|_{A}$. If $f^{\prime}$ is universal, as a map to $Y$, then $f$ must be universal. Thus, the behavior of $f$ on $X \backslash A$ may be completely arbitrary. In order to characterize universal maps on trees, we need to restrict the map to a subtree on which $f$ is "minimal universal".

Definition 2.6. Let $f: R \rightarrow S$ be universal map of trees. If the restriction of $f$ to any proper subtree of $R$ is not universal as a map to $S$, then we shall call $f$ a minimal universal map to $S$.

Theorem 2.7. If $f: R \rightarrow S$ is a universal map of trees, then there is a subtree $R^{*}$ of $R$ such that $\left.f\right|_{R^{*}}$ is a minimal universal map to $S$. Further, if $M$ denotes the middle space of $f$ and $l m$ is the monotone-light factorization of $f$, then $\left.l\right|_{m\left(R^{*}\right)}$ is minimal universal to $S$.

Proof. Let $R^{*}$ be the intersection of a maximal tower of subtrees $R_{\alpha}$ of $R$ such that for each $\alpha,\left.f\right|_{R_{\alpha}}$ is universal to $S$. Let $h=\left.f\right|_{R^{*}}$, and suppose that $h$ is not universal to $S$. Let $g: R^{*} \rightarrow S$ be a map which misses $h$. Now since $S$ is an absolute retract, there is a map $g^{\prime}: R \rightarrow S$ which extends $g$. Choose $\epsilon>0$ so that $d(h(x), g(x))>\epsilon$ for all $x \in R^{*}$. Choose $\delta>0$ so that if $x, y \in R$ with $d(x, y)<\delta$, then $d(f(x), f(y))<\epsilon / 3$ and $d\left(g^{\prime}(x), g^{\prime}(y)\right)<\epsilon / 3$. Now choose $R_{\alpha}$ so that each point of $R_{\alpha}$ is within $\delta$ of some point of $R^{*}$. We will show that $g^{\prime}$ misses $f$ on $R_{\alpha}$.

Let $x \in R_{\alpha}$. Choose $y \in R^{*}$ so that $d(x, y)<\delta$. Now $\epsilon<d(h(y), g(y))$ $\leq d(f(y), f(x))+d\left(f(x), g^{\prime}(x)\right)+d\left(g^{\prime}(x), g(y)\right)<2 \epsilon / 3+d\left(f(x), g^{\prime}(x)\right)$. Hence $d\left(f(x), g^{\prime}(x)\right)>0$ for all $x \in R_{\alpha}$. But this contradicts our assumption that $f$ restricted to $R_{\alpha}$ is universal. So $h: R^{*} \rightarrow S$ is universal.

Now since the tower is maximal it follows that $R^{*}$ is in the tower and $h: R^{*} \rightarrow S$ is minimal universal to $S$. The proof of the last statement follows from 2.5

\section{Combinatorial maps}

We are going to define a class of mappings between trees called combinatorial. Later, in Section 4, we will show that light minimal universal mappings must be combinatorial. First, we define the notion of subedge. 


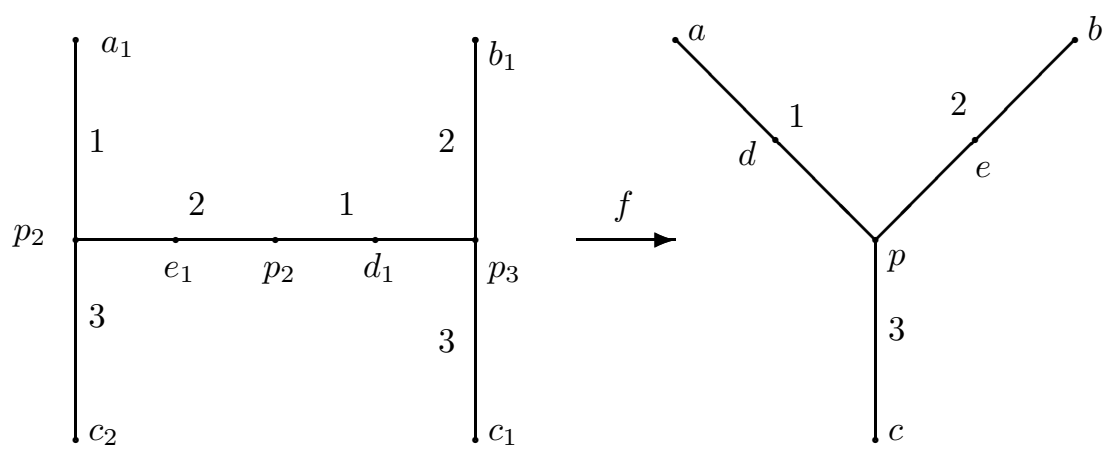

Figure 1. A combinatorial map from the $\mathrm{H}$ to the $\mathrm{Y}$.

Definition 3.1. Let $f: R \rightarrow S$ be a map of trees. An $\operatorname{arc} r s$ in $R$ is a subedge of $R$ provided that

i) $r s$ lies in an edge of $R$, and

ii) there is an edge $a b$ of $S$ so that $r s$ is a component of $f^{-1}(a b)$.

We really should call a subedge of $R$ an $f$-subedge of $R$, but it is always clear from the context which $f$ we are talking about. Now the definition of combinatorial can be stated.

Definition 3.2. A map $f: R \rightarrow S$ of trees is combinatorial provided that

i) $f^{-1}\left(E_{S}\right)=E_{R}$,

ii) each terminal edge of $R$ is a subedge of $R$,

iii) each point of $R$ lies in a subedge of $R$,

iv) no edge of $R$ contains two subedges which map to the same edge of $S$.

So, among other things, if $f: R \rightarrow S$ is a combinatorial map, then as $t$ traverses an edge of $R, f(t)$ cannot re-enter an edge of $S$ once it has left it.

In Figure 1, we describe a combinatorial map from the $\mathrm{H}$ to the $\mathrm{Y}$ which is simplicial. Points named $x_{i}$ in the domain are mapped to the points named $x$ in range, and the result is extended linearly on each of the subarcs. (Superimposed on this simplicial diagram are some numbers, which we describe shortly.)

Combinatorial maps have three additional properties of importance.

Lemma 3.3. Suppose $f: R \rightarrow S$ is a combinatorial map of trees. Then

v) $f$ is a surjection to $S$.

vi) There are only finitely many subedges of $R$.

vii) If $p$ is a branchpoint of $R$ of order $n$, then $f(p)$ is a branchpoint of $S$ of order at least $n$.

Proof. Let $x \in S$. Then there are endpoints $p, q \in S$ such that $x \in p q$. By 3.2 i) there are endpoints $p^{\prime}$ and $q^{\prime}$ in $R$ which map to $p, q$ respectively. Note that $x \in p q \subset f\left(p^{\prime} q^{\prime}\right)$ and so v) is established.

Let $a b$ be an edge of $S$. By 3.2 iv) there are no more subedges of $R$ mapping into $a b$ than there are edges of $R$. Hence there are only finitely many subedges mapping into $a b$. Since there are only finitely many edges in $S$, vi) is established.

Using iii) and vi), we see that each edge of $R$ is the union of finitely many subedges of $R$ and hence there are $n$ subedges of $R$ with $p$ as an endpoint. Using 


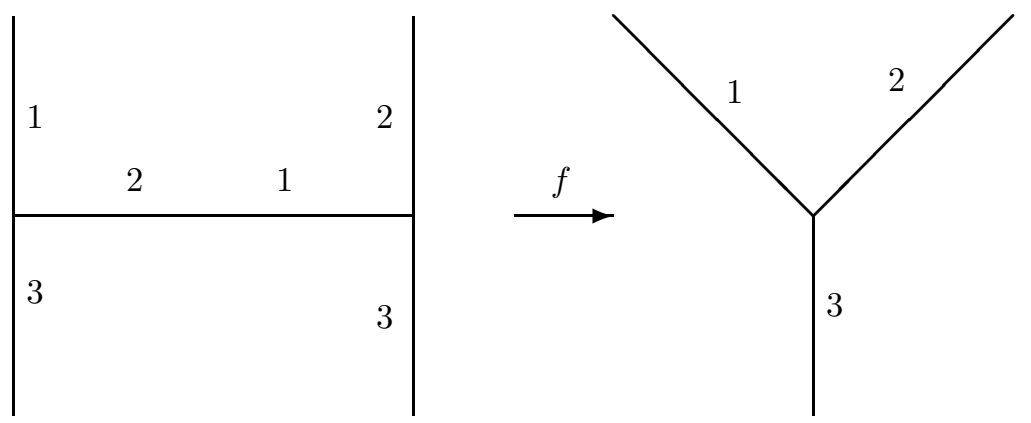

Figure 2. The edge-subedge diagram for the map in Figure 1.

iii) and the definition of subedge, no two of these subedges can map to the same edge of $S$. This establishes vii).

Edge-subedge diagrams. It turns out to be convenient to describe a combinatorial map by means of its edge-subedge diagram. To construct the edge-subedge diagram for a combinatorial map $f: R \rightarrow S$, first label all of the edges of $S, 1,2$, $\cdots, n$. Then label each subedge of $R$ with the label of the edge of $S$ to which it is mapped by $f$. Figure 1 shows the construction of the edge-subedge diagram superimposed on the simplicial diagram.

Several properties of this labeling are evident from the properties of a combinatorial map.

(i) No two subedges in the same edge of $R$ have the same label.

(ii) All the subedges around a branchpoint of $R$ have distinct labels.

(iii) Adjacent subedges of $R$ have labels belonging to adjacent edges of $S$.

(iv) Each terminal subedge of $R$ has only one label written on it, that label belongs to a terminal subedge of $S$, and each label belonging to a terminal edge of $S$ is written on at least one terminal edge of $R$.

In Figure 2, we have removed all but the labels from the picture, leaving the edge-subedge diagram of $f$.

Working from the labeling given in Figure 2, we can construct a combinatorial map which has Figure 2 as its edge-subedge diagram, by defining the map piecewise on each subedge and gluing the resulting maps together. For example, the map $f$ in Figure 2 maps each of the terminal edges of the $\mathrm{H}$ homeomorphically onto its corresponding terminal edge in $\mathrm{Y}$, taking the branchpoint to the branchpoint. Then the leftmost subedge on the horizontal edge of the $\mathrm{H}$ is folded into the 1-edge of the $\mathrm{Y}$ with both endpoints going to the branchpoint, being certain not to let the endpoint of the 1-edge be covered in the process. Similarly, the remaining subedge is folded into the 2-edge of the Y.

Each of the remaining figures in this paper gives an edge-subedge diagram of some map. Such diagrams do not completely determine the map, but rather an equivalence class of maps.

Edge-subedge diagrams which are diagrams of a combinatorial map from $R$ to $S$ can be constructed in advance. To do this, first label the edges of $S$ by 1,2 , etc., and then list sequences of these labels along each edge of $R$ in such a way 


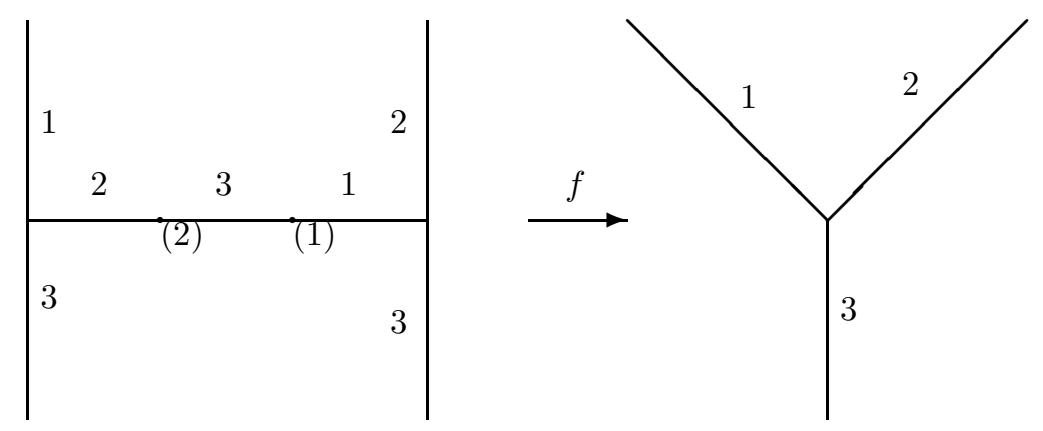

Figure 3. Combinatorial but not universal

that the labeling conditions (i)-(iv) given above are satisfied. When this is done, an equivalence class of combinatorial maps is determined.

For example, the edge-subedge diagram given in Figure 3 describes a combinatorial map $f$ from the $\mathrm{H}$ to the $\mathrm{Y}$ which is not universal. Included in the diagram are some labels in parentheses which describe how to construct a map $g: H \rightarrow Y$ which misses $f$. The range of $g$ is the arc in $S$ connecting the endpoint of leg 2 with the endpoint of leg 1 . Thus $g$ sends everything to the left of (2) in $\mathrm{H}$ to the endpoint of the 2 leg in Y, and $g$ sends everything to the right of (1) in $\mathrm{H}$ to the endpoint of the $1 \mathrm{leg}$ in Y. On the arc connecting (2) and (1) in $H, g$ is a homeomorphism to the arc connecting the endpoints of the 2 and 1 legs in $S$. One needs to choose the points (1) and (2) so that for $t \in(2)(1), f(t)$ lies in the 3 leg of $Y$ with the vertex removed. A $g$ constructed like this will miss $f$.

\section{Light Minimal UNiversal MAPS ARE COMBinAtorial}

The goal of this section is to prove Theorem 4.9. We first prove two lemmas.

Lemma 4.1. Let $f: R \rightarrow S$ be a universal map of trees. Suppose $R$ is the union of two branches $B_{1}$ and $B_{2}$ whose intersection is a single point $p$. If for each $i=1,2$, $g_{i}: B_{i} \rightarrow S$ is a map which misses $\left.f\right|_{B_{i}}$, then $f(p)$ lies in the arc from $g_{1}(p)$ to $g_{2}(p)$.

Proof. Suppose that $f(p) \notin g_{1}(p) g_{2}(p)$. Then we can choose a continuum $K$ containing $p$ in its interior so small that $f(K) \cap\left(g_{1}\left(B_{1} \cap K\right) \cup g_{1}(p) g_{2}(p) \cup g_{2}\left(B_{2} \cap K\right)\right)=$ $\emptyset$. Let $S^{\prime}=g_{1}\left(B_{1} \cap K\right) \cup g_{1}(p) g_{2}(p) \cup g_{2}\left(B_{2} \cap K\right)$. Since $S^{\prime}$ is a subtree of $S$, by the Tietze Extension Theorem, there is a map $h: K \rightarrow S^{\prime}$ which agrees with $g_{i}$ on the boundary of $K$ in $B_{i}$, for $i=1,2$. Note that $h$ misses $\left.f\right|_{K}$. But then the map $g$ defined to be the join of $h$ with $\left.g_{1}\right|_{\left(B_{1} \backslash K\right)}$ and $\left.g_{2}\right|_{\left(B_{2} \backslash K\right)}$ misses $f$, contradicting the universality of $f$.

Lemma 4.2. Let $f: R \rightarrow S$ be a minimal universal map of trees. Suppose that $x y$ is an arc contained in some nonterminal edge of $R$. Define $R_{1}=C_{y}(x)$ and $R_{2}=C_{x}(y)$. For $i=1,2$, let $g_{i}: R_{i} \rightarrow S$ be a map so that $g_{i}$ misses $\left.f\right|_{R_{i}}$ and $g_{i}$ is endpoint valued at $x$ and $y$. Then

(i) $f(x y) \subset g_{1}(x) g_{2}(y)$ (in particular, $f(x y)$ is an arc or a point), and

(ii) either $f(x y)$ lies in an edge of $S$ or $g_{1}(x)<f(x) \leq f(y)<g_{2}(y)$ in that order on $g_{1}(x) g_{2}(y)$. 
Proof. First, note that, by 4.1, for each $\left.t \in x y, f(t) \in g_{1}(t) g_{2}(t)\right)$, hence $f(t)$ is not an endpoint of $S$. Thus $f(x y)$ contains no endpoint of $S$. We make use of this in the proofs of both parts of the lemma.

If (i) fails, then there exist $r, s \in x y$, with $x<r<s<y$ such that $f(r s) \cap$ $g_{1}(x) g_{2}(y)=\emptyset$.

Define a map $h: x y \rightarrow g_{1}(x) g_{2}(y)$ by $h(x r)=\left\{g_{1}(x)\right\}, h(s y)=\left\{g_{2}(y)\right\}$, and $h$ maps $r s$ homeomorphically onto $g_{1}(x) g_{2}(y)$ with $h(r)=g_{1}(x), h(s)=g_{2}(y)$. We claim that $h$ misses $f$ on $x y$. To see this, we have by construction that $f(r s) \cap$ $g_{1}(x) g_{2}(y)=\emptyset$. So $h$ misses $f$ on $r s$. But also $f(x y) \cap\left\{g_{1}(x), g_{2}(y)\right\}=\emptyset$, since $f(x y)$ contains no endpoint of $S$ (from the note at the beginning of the proof), and $g_{1}(x), g_{2}(y)$ are endpoints of $S$. So $h$ misses $f$ on $x r$ and $s y$. This establishes the claim.

Define $g_{1}^{\prime}$ to be $g_{1}$ restricted to $\left(R_{1} \backslash x y\right) \cup\{x\}$ and $g_{2}^{\prime}$ to be $g_{2}$ restricted to $\left(R_{2} \backslash x y\right) \cup\{y\}$. Then define $g: R \rightarrow S$ to be the join of $g_{1}^{\prime}, h$, and $g_{2}^{\prime}$. Then $g$ misses $f$, a contradiction. This proves (i).

To prove (ii), assume $f(x y)$ does not lie in an edge of $S$. By part (i), $f(x y)$ is an arc $a b$ contained in $g_{1}(x) g_{2}(y)$, ordered so that $g_{1}(x)<a<b<g_{2}(y)$. Then there is a branchpoint $c$ of $S$ lying in the interior of $a b$ and an endpoint $e$ of $S$ such that $e c \cap a b=\{c\}$.

Assume that (ii) fails. We will construct a map on $R$ which misses $f$. Since (ii) fails, then $f(y)<f(x)$ on the arc $g_{1}(x) g_{2}(y)$, and hence $g_{1}(x)<a \leq f(y)<f(x) \leq$ $b<g_{2}(y)$.

To define the map, we first choose points $x^{\prime}, x^{\prime \prime}, y^{\prime}$, and $y^{\prime \prime}$ in $x y$ so that $x \leq$ $x^{\prime}<x^{\prime \prime}<y^{\prime \prime}<y^{\prime} \leq y$, and $f\left(x^{\prime} x^{\prime \prime}\right) \subset(c b \backslash\{c\}), f\left(y^{\prime} y^{\prime \prime}\right) \subset(a c \backslash\{c\})$.

There are three cases to consider:

(1) If $f(y)<c<f(x)$, choose $x^{\prime}=x, y^{\prime}=y$, and $x^{\prime \prime}, y^{\prime \prime}$ so close to $x^{\prime}$ and $y^{\prime}$ respectively that $f\left(x^{\prime} x^{\prime \prime}\right) \subset c b \backslash\{c\}$ and $f\left(y^{\prime} y^{\prime \prime}\right) \subset c a \backslash\{c\}$.

(2) If $c \leq f(y)<f(x)$, choose $x^{\prime}=x$ and $x^{\prime \prime}$ as above. Then, since $c$ lies in the interior of $f(x y)$, we can choose $y^{\prime \prime}$ and $y^{\prime}$ so that $x^{\prime \prime}<y^{\prime \prime}<y^{\prime}<y$ and $f\left(y^{\prime \prime} y^{\prime}\right) \subset c a \backslash\{c\}$.

(3) If $f(y)<f(x) \leq c$, choose $y^{\prime}=y$ and $y^{\prime \prime}$ as in case 1 and choose $x^{\prime}$ and $x^{\prime \prime}$ as in case 2 .

Now define a map (see Figure 4) $h: x y \rightarrow g_{1}(x) g_{2}(y) \cup e c$ as follows: $h$ is constant on the three subintervals $x x^{\prime}, x^{\prime \prime} y^{\prime \prime}$, and $y^{\prime} y$, namely $h\left(x x^{\prime}\right)=\left\{g_{1}(x)\right\}, h\left(x^{\prime \prime} y^{\prime \prime}\right)=$ $\{e\}$, and $h\left(y^{\prime} y\right)=\left\{g_{2}(y)\right\}$. Note that $h$ misses $f$ on these subintervals since $h$ is endpoint valued there and $f$ never assumes an endpoint value there (use the note at the beginning of proof). Define $h$ on $x^{\prime} x^{\prime \prime}$ so that it maps $x^{\prime} x^{\prime \prime}$ homeomorphically onto $g_{1}(x) c \cup c e$ (taking $x^{\prime}$ to $g_{1}(x)$ and $x^{\prime \prime}$ to $e$ ) and $y^{\prime \prime} y^{\prime}$ homeomorphically onto $e c \cup c g_{2}(y)$ (taking $y^{\prime \prime}$ to $e$ and $y^{\prime}$ to $g_{2}(y)$ ). By the way $x^{\prime} x^{\prime \prime}$ and $y^{\prime} y^{\prime \prime}$ were chosen, $f$ and $h$ never coincide on these arcs. Hence the map $g$ on $R$ which is the join of $\left.g_{1}\right|_{R_{1} \backslash x y},\left.g_{2}\right|_{R_{2} \backslash x y}$, and $h$ misses $f$, a contradiction to the universality of $f$.

This completes the proof of (ii).

The proof of Theorem 4.9 is broken into several propositions. In each of 4.3, 4.4, $4.5,4.6,4.7$, and $4.8, f: R \rightarrow S$ is assumed to be a light minimal universal map of trees.

Proposition 4.3. If $p \in R$, then $\operatorname{ord}_{R}(p) \leq \operatorname{ord}_{S}(f(p))$. 


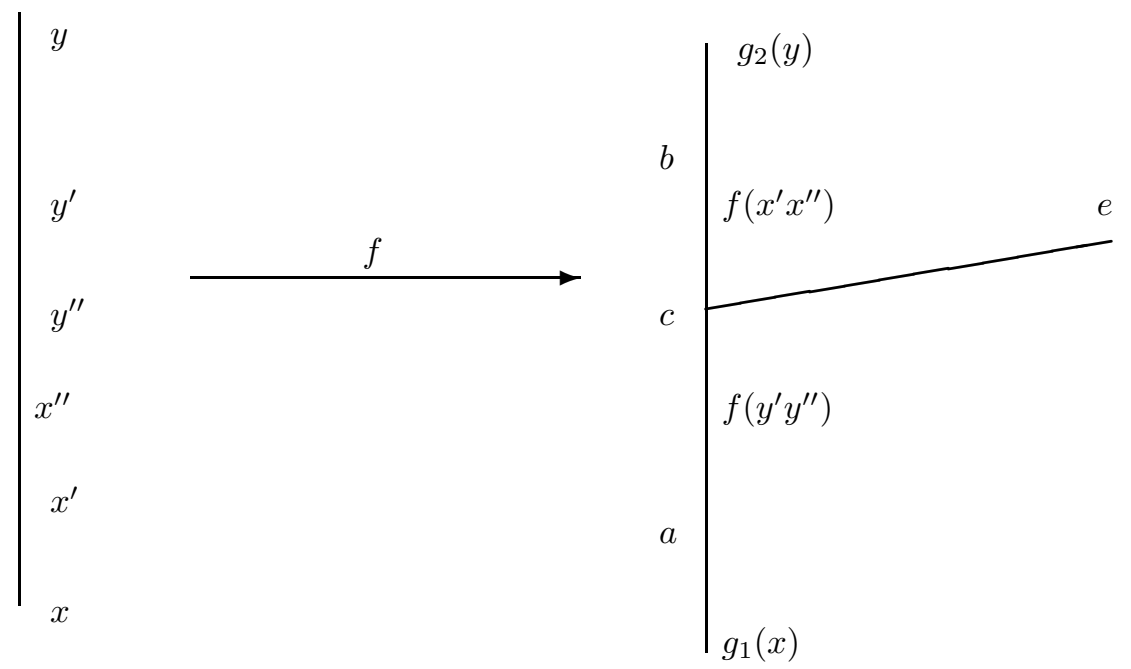

FiguRE 4. Diagram for Lemma 4.2

Proof. Let $n=\operatorname{ord}_{R}(p)$ and $m=\operatorname{ord}_{S}(f(p))$. We can assume $n>1$. Let $A_{1}, \cdots, A_{n}$ denote the closures of the components of $R \backslash\{p\}$, and let $R_{i}$ be the closure of the complement of $A_{i}$. Also let $D_{1}, \cdots, D_{m}$ denote the closure of the components of $S \backslash\{f(p)\}$. Since $f$ is minimal universal there are, by Lemma 2.2, maps $g_{i}: R_{i} \rightarrow S, i=1, \cdots, n$, such that $g_{i}$ misses $\left.f\right|_{R_{i}}$ and $g_{i}(p) \in E_{S}$. Suppose for the moment that $m<n$. Then for some $i, j, k$ with $i \neq j$ it must be that $g_{i}(p)$, $g_{j}(p) \in D_{k}$ and so $f(p) \notin g_{i}(p) g_{j}(p)$. However, we can apply Lemma 4.1 with $B_{1}=R_{i}, B_{2}=A_{i}, g_{1}=g_{i}$, and $g_{2}=\left.g_{j}\right|_{A_{i}}$ to conclude that $f(p) \in g_{i}(p) g_{j}(p)$.

This contradiction shows that $m \geq n$.

Proposition 4.4. If $a b$ is an edge of $S$, then each component of $f^{-1}(a b)$ is contained in some edge of $R$. Hence each nondegenerate component of $f^{-1}(a b)$ is a subedge of $R$.

Proof. Suppose not. Then there is an arc $x y$ in $f^{-1}(a b)$ and a branchpoint $p$ of $R$ in $x y$ such that $x p$ and $p y$ are arcs, lying in different edges of $R$. Also since $p$ is a branchpoint of $R$, we can choose $z \in R$ so that the arc $p z$ meets the arc $x y$ only in the point $p$. Let $R_{1}, R_{2}$ and $R_{3}$ denote the closure of the complement of $C_{p}(x), C_{p}(y)$, and $C_{p}(z)$, respectively. Since $f$ is minimal universal, there are maps $g_{i}: R_{i} \rightarrow S, i=1,2,3$, which miss $f_{\mid R_{i}}$, and are endpoint valued at $p$. At least two of the endpoints $g_{i}(p)$ must lie in one of the trees $C_{a}(b)$ and $C_{b}(a)$. Without loss of generality, assume that that $g_{1}(p)$ and $g_{2}(p)$ both lie in $C_{a}(b)$. Hence, $g_{1}(p) g_{2}(p) \cap a b \subset\{b\}$.

Now $f(p)$ is a branchpoint of $S$ in $a b$, so $f(p)=a$ or $f(p)=b$. By Lemma 4.1, $f(p) \in g_{1}(p) g_{2}(p)$ and so $f(p)=b$. Now by the continuity of $g_{2}$ and the lightness of $f$, we can choose $t \in x p$ so close to $p$ that $g_{2}(t) g_{1}(p) \cap a b \subset\{b\}$ and $f(t) \neq b$. Thus $f(t)$ is not in the arc $g_{1}(p) g_{2}(t)$. But we can extend the domain of $g_{1}$ to include $t p$ by defining $g_{1}(t p)=\left\{g_{1}(p)\right\}$. However, now $f(t) \notin g_{1}(t) g_{2}(t)$ and this contradicts Lemma 4.1. 
Proposition 4.5. $f^{-1}\left(E_{S}\right)=E_{R}$. Furthermore, if $e \in E_{R}$ and $b \in B_{R}$ with eb a terminal edge of $R$, then $f(e b)$ is a terminal edge of $S$. In fact, each terminal edge of $R$ is a subedge of $R$.

Proof. That $f^{-1}\left(E_{S}\right) \subset E_{R}$ follows immediately from Proposition 4.3. Conversely, suppose $e \in E_{R}$ but $f(e) \notin E_{S}$. Then choose $t \in e b \backslash\{e\}$ so close to e that $f(e t) \cap E_{S}=\emptyset$. Since $f$ is minimal universal there is a map $g^{\prime}: C_{t}(b) \rightarrow S$ such that $g^{\prime}$ misses $\left.f\right|_{C_{t}(b)}$ and $g^{\prime}(t) \in E_{S}$. Now the map $g: R=C_{t}(b) \cup e t \rightarrow S$ given by $g(x)=g^{\prime}(x)$ if $x \in C_{t}(b)$ and $g(x)=g^{\prime}(t)$ if $x \in$ et clearly misses $f$, a contradiction. Hence $E_{R} \subset f^{-1}\left(E_{S}\right)$.

Now suppose that $e b$ is a terminal edge of $R$. Then $f(e)$ is an endpoint of $S$. Label $b^{\prime} \in B_{S}$ so that $f(e) b^{\prime}$ is a terminal edge of $S$. If $f(e b) \not \subset f(e) b^{\prime}$, then we can choose $t \in e b$, with $t \neq b$, such that $f(t) \notin f(e) b^{\prime}$. Since $f$ is minimal universal there is, by Lemma 2.2, a map $g_{1}: C_{t}(b) \rightarrow S$ such that $g_{1}$ misses $f \mid C_{t}(b)$ and $g_{1}(t) \in E_{S}$.

We have already proved that $f^{-1}\left(E_{S}\right)$ is a subset of $E_{R}$. It follows that $f(t e) \cap$ $E_{S}=\{f(e)\}$. We claim that $g_{1}(t)=f(e)$. If not, then $g_{1}(t) \notin f(t e)$ and we can extend $g_{1}$ to all of $R$ by defining $g_{1}(e t)=\left\{g_{1}(t)\right\}$. This extension misses $f$, contradicting the universality of $f$, and establishing the claim.

Choose $d \in E_{S}$ such that $d \neq f(e)$ and $f(t) \notin d g_{1}(t)$. Define $g_{2}:$ et $\rightarrow S$ to be the constant map with value $d$. Since $f(e t) \cap E_{S}=\{f(e)\}$ and $d \neq f(e), g_{2}$ misses $\left.f\right|_{e t}$. But now 4.1 is contradicted (take $p=t, B_{1}=C_{t}(b), B_{2}=e t$ ).

Hence $f(e b) \subset f(e) b^{\prime}$. By $4.3, f(b) \in B_{S}$, and so $f(b)=b^{\prime}$. It follows that $f(e b)=f(e) b^{\prime}$ is a terminal edge of $S$. That $e b$ is in fact a subedge of $R$ now follows from Proposition 4.4: the component of $f^{-1}\left(f(e) b^{\prime}\right)$ containing $e b$ is contained in an edge and hence is $e b$.

Proposition 4.6. No edge of $R$ contains two subedges which map to the same edge in $S$.

Proof. Suppose not. Then there is an edge $a b$ of $S$, an edge $p q$ of $R$ and three points $x_{1}, x_{2}$, and $x_{3}$ in $p q$ so that $p<x_{1}<x_{2}<x_{3}<q$, and $f\left(x_{1}\right)$ and $f\left(x_{3}\right)$ both lie in the interior of $a b$, and $f\left(x_{2}\right)$ lies in the interior of some edge of $S$ sharing an endpoint with $a b$, which we will assume without loss of generality to be $b$. Let $T_{1}=C_{x_{3}}\left(x_{1}\right)$ and $T_{2}=C_{x_{1}}\left(x_{3}\right)$. Since $f$ is minimal universal, there are maps $g_{i}: T_{i} \rightarrow S$ which miss $\left.f\right|_{T_{i}}$ and are endpoint-valued at $x_{1}, x_{2}$ and $x_{3}$.

We will apply Lemma 4.2 successively to the $\operatorname{arcs} x_{1} x_{2}, x_{1} x_{3}$, and $x_{2} x_{3}$. We can do this because $p q$ is not a terminal edge (by 4.5, since its image is not an edge).

Apply Lemma 4.2, with $x y=x_{1} x_{2}$. By (i), $f\left(x_{1} x_{2}\right) \subset g_{1}\left(x_{1}\right) g_{2}\left(x_{2}\right)$, and by (ii), $g_{1}\left(x_{1}\right)<a<f\left(x_{1}\right)<b<f\left(x_{2}\right)<g_{2}\left(x_{2}\right)$ in the natural order on $g_{1}\left(x_{1}\right) g_{2}\left(x_{2}\right)$.

Again by Lemma 4.2(i),

$$
f\left(x_{1} x_{3}\right) \subset g_{1}\left(x_{1}\right) g_{2}\left(x_{3}\right) .
$$

So $f\left(x_{1}\right)$ is in the $\operatorname{arc} g_{1}\left(x_{1}\right) g_{2}\left(x_{3}\right)$, and it follows that, for $i=1,3, g_{1}\left(x_{1}\right)<$ $a<f\left(x_{i}\right)<b<f\left(x_{2}\right)<g_{2}\left(x_{3}\right)$ in the natural order on $g_{1}\left(x_{1}\right) g_{2}\left(x_{3}\right)$. Thus $b \notin f\left(x_{2}\right) g_{2}\left(x_{3}\right)$.

But now, by Lemma 4.2(i), $f\left(x_{2} x_{3}\right) \subset g_{1}\left(x_{2}\right) g_{2}\left(x_{3}\right)$ and by Lemma 4.2(ii), $g_{1}\left(x_{2}\right)<f\left(x_{2}\right) \leq f\left(x_{3}\right)<g_{2}\left(x_{3}\right)$ in the natural order on $g_{1}\left(x_{2}\right) g_{2}\left(x_{3}\right)$. Since $b$ lies between $f\left(x_{2}\right)$ and $f\left(x_{3}\right)$, we get $f\left(x_{2}\right)<b<f\left(x_{3}\right)<g_{2}\left(x_{3}\right)$. Thus $b \in f\left(x_{2}\right) g_{2}\left(x_{3}\right)$ which contradicts the end of the preceding paragraph. This completes the proof. 
Corollary 4.7. The set of subedges of $R$ is finite.

Proof. By 4.6 there are no more subedges of $R$ mapping to a particular edge of $S$ than there are edges in $R$. Since both $R$ and $S$ have only finitely many edges, it follows that there are only finitely many subedges of $R$.

Proposition 4.8. Each point of $R$ lies in a subedge of $R$.

Proof. Let $R^{\prime}$ be the union of the nondegenerate components of the sets $f^{-1}(a b)$, as $a b$ runs over the edges of $S$. Then by 4.4 and 4.7 , there are only finitely many such components and so $R^{\prime}$ is closed. If $R-R^{\prime}$ is nonempty, then there is a nondegenerate $\operatorname{arc} A$ in $R-R^{\prime}$ Since $f$ is light, the image $f(A)$ is nondegenerate, and hence $A$ contains a nondegenerate subarc $B$ such that $f(B)$ lies in an edge of $S$. This is a contradiction, and so $R-R^{\prime}$ is empty.

Now we can prove that light minimal universal maps are combinatorial.

Theorem 4.9. If $f: R \rightarrow S$ is a light minimal universal map of trees, then $f$ is combinatorial.

Proof. Properties i) and ii) of Definition 3.2 are established by 4.5. Property iii) is established in 4.8. Property iv) is established in 4.6.

\section{Further PROPERTIES OF Light Minimal UNIVERSAL MAPS}

We have already seen that light minimal universal maps are combinatorial. In this section, we derive three additional properties of minimal universal maps (Theorems 5.3, 5.7, and 5.8). In the next section, these additional properties are used to characterize universal maps on trees.

Definition 5.1. Let $f: R \rightarrow S$ be a combinatorial map of trees. A subedge $a b$ of $R$ is called a folded subedge if $f(a)=f(b)$. We say that the folded subedges of $R$ are paired if each edge of $R$ which contains a folded subedge contains exactly two folded subedges, each of which contains an endpoint of the edge.

The answer to the question of determining which combinatorial maps on $R$ are universal hinges on the distribution of the folded subedges of $R$.

The following lemma is a stronger version of Lemma 2.2 which we use repeatedly in establishing properties of the folded subedges of $R$.

Lemma 5.2. Suppose that $f: R \rightarrow S$ is a light minimal universal map of trees, and $F$ is a finite subset of $R$. Let $b$ be a branchpoint of $R$, let $a$ be a point in a subedge of $R$ containing $b$ with $f(a) \neq f(b)$ and let $e$ be a point of $E_{S} \backslash C_{f(b)}(f(a))$. Then there is a map $g: C_{b}(a) \rightarrow S$ such that $g$ is endpoint valued on $F \cap C_{b}(a), g$ misses $\left.f\right|_{C_{b}(a)}$, and $g(b)=e$.

Proof. Suppose that $\operatorname{ord}_{R}(b)=n$. Let $s_{1}, \cdots, s_{n}$ be the vertices of $R$ adjacent to $b$. Since $b$ is a branchpoint, $n>2$. Without loss of generality, we may assume that $a \in C_{b}\left(s_{1}\right)$.

For $i=2,3$, let $P_{i}=\overline{R \backslash C_{b}\left(s_{i}\right)}$. Then $P_{i}$ is a proper subcontinuum of $R$ containing $C_{b}(a)$. So, by 2.2 , there is a map $g_{i}: P_{i} \rightarrow S$ which is endpoint valued on $\{a, b\} \cup\left(F \cap P_{i}\right)$ and misses $\left.f\right|_{P_{i}}$.

By $4.1, f(b) \in g_{2}(b) g_{3}(b)$, so one of $g_{2}(b)$ and $g_{3}(b)$ is not in $C_{f(b)}(f(a))$. Without loss of generality we can assume that $g_{2}(b) \notin C_{f(b)}(f(a))$. Choose $x \in b a$ with $f(x) \neq f(b)$ so close to $b$ that $g_{2}(x b) \cap C_{f(b)}(f(a))=\emptyset$. Now choose $y \in b x \backslash\{x\}$ 
so that $f(x y) \subset C_{f(b)}(f(a)) \backslash\{f(b)\}$. Note that $f(x y) \cap g_{2}(x) e=\emptyset$. Then let $m: x b \rightarrow g_{2}(x) e$ be any map with $m(x)=g_{2}(x)$ and $m(y b)=\{e\}$. By construction, $m$ misses $\left.f\right|_{b x}$. So defining $g$ to be the join of $m$ with $\left.g_{2}\right|_{C_{x}(a)}$ establishes the lemma.

Theorem 5.3. If $f: R \rightarrow S$ is a light minimal universal map of trees, then the folded subedges of $f$ are paired.

The proof of this theorem is contained in the next three propositions. In each one, $f: R \rightarrow S$ is assumed to be a light minimal universal map of trees.

Proposition 5.4. No edge which is a subedge is folded.

Proof. Suppose to the contrary, that there are edges $p q$ and $c d$ of $R$ and $S$ respectively so that $f(p q) \subset c d, f(p)=f(q)=c$. Now $p q$ is not a terminal edge. For suppose it is. Then one of $p$ and $q$ is an endpoint of $R$ and hence $c$ is an endpoint of $S$ by Theorem 4.9. But then both $p$ and $q$ are endpoints of $R$ by 4.9 , and so $R=p q$. But then $S=c d$ and some non endpoint of $p q$ must go to $d$ under $f$. This contradicts 4.9 , and so $p q$ is not terminal.

So $C_{p}(q)$ and $C_{q}(p)$ are proper subcontinua of $R$, and there are maps $g_{1}: C_{p}(q) \rightarrow$ $S$ and $g_{2}: C_{q}(p) \rightarrow S$ which miss $f$ on their domain. We can assume that $g_{1}$ and $g_{2}$ are endpoint valued at $p, q$, and at a point $t$ in $p q$ chosen so that $f(p q)=f(p) f(t)$.

Now the arc $g_{1}(p) g_{2}(p)$ must contain $c=f(p)$ by 4.1. In fact, it must contain $c d$, for if not then $g_{1}(p) g_{2}(p) \cap c d=\{c\}$ and by choosing $x \in p q$ close to $p$ so that $f(x) \notin g_{1}(p) g_{2}(p)$ and $g_{1}(x) g_{2}(x) \subset g_{1}(p) g_{2}(p)$ we see that 4.1 is violated. In the same way $g_{1}(q) g_{2}(q)$ contains $c d$.

Also the arcs $g_{1}(p) g_{1}(q)$ and $g_{2}(p) g_{2}(q)$ must each intersect $c d$ at most in $c$ or in $d$, by 1.2 .

Now one of two cases must occur:

If $g_{1}(p)<c<d \leq g_{2}(p)$, then apply 5.2 with $b=q, a=t$, and $e=g_{1}(q)$ to obtain a map $g: C_{q}(t) \rightarrow S$ which misses $f$ on $C_{q}(t)$ and has the value $g(q)=g_{1}(q)$. Now the map $h$ which is the join of $g$ and $g_{1}$ restricted to $C_{p}(q) \backslash p q$ misses $f$ and a contradiction is obtained.

If $g_{2}(p)<c<d \leq g_{1}(p)$, then apply 5.2 with $b=p, a=t$, and $e=g_{2}(p)$ to obtain a map $g: C_{p}(t) \rightarrow S$ which misses $f$ on $C_{p}(t)$ and has the value $g(p)=g_{2}(p)$. Now the map $h$ which is the join of $g$ and $g_{2}$ restricted to $C_{q}(p) \backslash p q$ misses $f$ and a contradiction is obtained.

Since each case leads to a contradiction, our initial supposition is false and the theorem is established.

Proposition 5.5. Each folded subedge contains exactly one endpoint of the edge in which it lies.

Proof. By 5.4, no folded subedge contains both endpoints of the edge in which it lies, so if the theorem fails, there are three subedges $r s, s t$, and $t u$ lying in an edge $p q$ of $R$, with $f(s)=f(t)=a$ and $f(s t)$ contained in an edge $a b$ of $S$. Since $f$ is combinatorial, $f(r u)$ is a triod with vertex $a$. This contradicts 4.2 , which says that $f(r u)$ is an arc.

Note that 5.5 says that no edge in $R$ contains more than two folded subedges. In fact,

Proposition 5.6. Each edge of $R$ contains none or two folded subedges. 
Proof. If not, there is an edge $p q$ of $R$ containing exactly one folded subedge $p r$, and an edge $c d$ of $S$ such that $f(p)=f(r)=c$ and $f(p r) \subset c d$. By 5.5, $r \neq q$. Let $s q$ be the subedge of $p q$ containing $q$. Then $f(s q)$ is an edge of $S$ with endpoints $f(s)=b$ and $f(q)=a$. Since $f$ is combinatorial, $p q$ is not a terminal edge and $C_{p}(q), C_{q}(p)$ are proper subsets of $R$. Since $f$ is minimal universal there are maps $g_{1}: C_{q}(p) \rightarrow S$ and $k: C_{p}(q) \rightarrow S$ which miss $f$ on their respective domains and are endpoint valued at $q$.

By 4.2, parts (i) and (ii), we have $f(p q) \subset g_{1}(p) k(q)$ and $g_{1}(p)<f(p) \leq f(q)<$ $k(q)$. We claim that $f(p) \neq f(q)$. For suppose $f(p)=f(q)$. Then $f(q)=f(r)$ and since $s q$ is not folded $f(s) \neq f(q)$. Hence $f(s) \neq f(r)$. But $f(r s) \supset f(r) f(s)$ and $f(r) f(s)=f(q) f(s)$ and $f(q) f(s)$ is an edge of $S$. So $r s$ contains a subedge mapping to $f(q) f(s)$. This contradicts 4.9 (no edge of a combinatorial map contains two subedges mapping to the same edge). So our claim that $f(p) \neq f(q)$ is established.

So $k(q) \in E_{S} \backslash C_{f(q)}(f(p))$. Now apply 5.2 with $b=q, a=s$ and $e=k(q)$ to obtain a map $g_{2}: C_{q}(p) \rightarrow S$ such that $g_{2}(q)=k(q)$ and $g_{2}$ misses $f$ on $C_{q}(p)$. But now the map $g$ which is the join of $g_{2}$ and $k$ restricted to $C_{p}(q) \backslash p q$ misses $f$ on $R$, a contradiction to the assumption that $f$ is universal. This establishes the theorem.

Now Theorem 5.3 follows from 5.5 and 5.6.

Theorem 5.7. If $f: R \rightarrow S$ is a light minimal universal map of trees, then each edge of $R$ which is not a subedge contains a folded subedge.

Proof. Suppose not. Let $p q$ be an edge of $R$ which is not a subedge but contains no folded subedge. Label the endpoints of the subedges of $p q$ in order from $p$ to $q$, $p=x_{0}<x_{1}<\cdots<x_{n}=q$. Since $p q$ is not a subedge, $n>1$. Now $f(p q)$ is an arc by 4.2 , (i).

We claim that $f(p q)=f(p) f(q)$ and that in the order from $f(p)$ to $f(q), f\left(x_{i}\right)<$ $f\left(x_{i+1}\right)$ for $i=0, \cdots, n-1$. Since $p q$ contains no folded subedge, $f\left(x_{i} x_{i+1}\right)=$ $f\left(x_{i}\right) f\left(x_{i+1}\right)$, for $i=0, \cdots, n-1$. Also, since $f$ is combinatorial, no two subedges of $p q$ map into to the same edge of $S$, and so $f\left(p x_{i}\right) \cap f\left(x_{i} q\right)=\left\{f\left(x_{i}\right)\right\}$ for $i=$ $1, \cdots, n-1$. Thus $f\left(x_{i}\right)$ is between $f\left(x_{j}\right)$ and $f\left(x_{k}\right)$ for $0 \leq j<i<k \leq n$ in the $\operatorname{arc} f(p q)$ for $i=1, \cdots, n-1$. Hence

$$
f(p)=f\left(x_{0}\right)<f\left(x_{1}\right)<\cdots<f\left(x_{n}\right)=f(q),
$$

and this establishes the claim.

By 4.5, $p q$ is not a terminal edge of $R$, so $C_{p}(q)$ and $C_{q}(p)$ are proper subcontinua of $R$. Choose an endpoint $e \notin C_{f(q)}(f(p))$ and apply 5.2 with $b=q$ and $a=x_{n-1}$ to obtain a map $g_{1}: C_{q}(p) \rightarrow S$ which is endpoint valued at $p$, misses $\left.f\right|_{C_{q}(p)}$, and $g_{1}(q)=e$.

Let $g_{2}: C_{p}(q) \rightarrow S$ be any map which is endpoint valued on $\{p, q\}$ and misses $f \mid C_{p}(q)$.

By 4.2 (ii), we have the inequality $g_{1}(p)<f(p)<f(q)<g_{2}(q)$. But, since $g_{1}(q)=e \notin C_{f(q)}(f(p))$, we have the inequality $g_{1}(p)<f(p)<f(q)<g_{1}(q)$. Using the inequality established above, we have

$$
g_{1}\left(x_{0}\right)<f\left(x_{0}\right)<f\left(x_{1}\right)<\cdots<f\left(x_{n}\right)<g_{1}\left(x_{n}\right) .
$$

So $g_{1}\left(x_{0}\right) \in C_{f\left(x_{1}\right)}\left(f\left(x_{0}\right)\right)$. Then, by $1.2, g_{1}\left(x_{1}\right) \in C_{f\left(x_{2}\right)}\left(f\left(x_{1}\right)\right)$. Continue to apply 1.2 to obtain $g_{1}\left(x_{i}\right) \in C_{f\left(x_{i+1}\right)}\left(f\left(x_{i}\right)\right)$ until $i=n-1$. But we have 
$g_{1}\left(x_{n}\right) \in C_{f\left(x_{n-1}\right)}\left(f\left(x_{n}\right)\right)$, and so $g_{1}$ stretches $x_{n-1} x_{n}$ over its $f$-image, a violation of 1.2. A contradiction has been reached and the theorem must hold.

Theorem 5.8. If $f: R \rightarrow S$ is a light minimal universal map of trees and $p$ is a branchpoint of order $n$ in $R$, then $f(p)$ is a branchpoint of order $n$ in $S$.

Proof. By 3.3, the order of $f(p) \geq n$. Label the subedges which contain $p, p s_{i}$, $i=1, \cdots, n$. In each of these subedges choose a point $x_{i}$ so that $f(p) \neq f\left(x_{i}\right)$. If the theorem is false, then there is an endpoint $e$ of $S$ such that $f\left(p x_{i}\right) \cap f(p) e=\{f(p)\}$ for all $i=1, \cdots, n$. Note that $e \notin C_{f(p)}\left(f\left(x_{i}\right)\right)$ for each $i$. Hence by Lemma 5.2, there are maps $g_{i}: C_{p}\left(x_{i}\right) \rightarrow S$ missing $f$ such that $g_{i}(p)=e$. The join of the maps $g_{i}$ misses $f$. This contradicts the universality of $f$ and so the theorem is proved.

Combining Theorems 4.9, 5.3, 5.7, and 5.8, we have the following result.

Theorem 5.9. Let $f: R \rightarrow S$ be a light minimal universal map of trees. Then $f$ is combinatorial and

(i) the folded subedges of $R$ are paired,

(ii) each edge of $R$ which is not a subedge contains a folded subedge, and

(iii) if $p$ is a branchpoint of order $n$ in $R$, then $f(p)$ has order $n$ in $S$.

\section{The CHARACTERIZATION}

The characterization is given in Theorem 6.3. We begin by showing that the three conditions of Theorem 5.9 are sufficient to establish that a combinatorial map of trees is universal. Note that $f$ is not assumed to be a light mapping in this theorem.

Theorem 6.1. Let $f: R \rightarrow S$ be a combinatorial map of trees such that

(i) the folded subedges of $R$ are paired,

(ii) each edge which is not a subedge contains a folded subedge,

(iii) if $p$ is a branchpoint of order $n$ in $R$, then $f(p)$ has order $n$ in $S$.

Then $f$ is universal.

Proof. Suppose $f$ is not universal. Then define sets $\mathcal{E}$ and $F$ as follows.

In each folded subedge $p r$ of $R$, with branchpoint $p$, let $x$ be the first point of $p r$ (in the order from $p$ to $r$ ) such that $f(p x)=f(p) f(x)=f(p r)=f(x r)=f(x) f(r)$. We will call the $\operatorname{arc} p x$ a half folded subedge. Let $\mathcal{E}$ be the (finite) set consisting of all the nonfolded subedges of $R$ together with all the half folded subedges formed above. Let $F$ be the set of endpoints of the $\operatorname{arcs}$ in $\mathcal{E}$, together with the endpoints of the subedges of $R$.

Now let $g: R \rightarrow S$ be a map which misses $f$ and is endpoint valued on the set $F$. Denote by $\mathcal{C}$ the set of all branches $C_{b}(r)$, where $b$ is a branchpoint of $R, b r \in \mathcal{E}$, and $g(b) \in C_{f(b)}(f(r))$.

First we show that $\mathcal{C}$ is not empty. To see this, let $b$ be any branch point of $R$. Since $f$ is combinatorial and iii) holds, there is a $b r \in \mathcal{E}$ such that $f(b r)$ is a subset of $f(b) g(b)$. Thus $g(b) \in C_{f(b)}(f(r))$, and so $C_{b}(r) \in \mathcal{C}$. So $\mathcal{C}$ is not empty.

Now we show that $\mathcal{C}$ is empty. To see this, note first that $\mathcal{C}$ is finite and hence it contains an element $C_{b}(r)$ which is minimal in the sense that if $b^{\prime} r^{\prime} \in \mathcal{E}$ with $C_{b^{\prime}}\left(r^{\prime}\right)$ properly contained in $C_{b}(r)$, then $g\left(b^{\prime}\right) \notin C_{f\left(b^{\prime}\right)}\left(f\left(r^{\prime}\right)\right)$.

Let $b a$ be the edge containing $b r$, and let $n=\operatorname{ord}_{R}(a)$. There are points $s_{i}$, $i=1, \cdots, n$, so that $a s_{i} \in \mathcal{E}$ and $s_{1} \in b a$.

There are two cases to consider. 
Case 1. ba contains no folded subedge.

In this case, by ii), $b a$ is a subedge of $R, s_{1}=b$, and $r=a$. So $f(b a)=f(b) f(a)$ is an edge of $S$. Since $g(b) \in C_{f(b)}(f(a))$, it follows from 1.2 that $g(a) \in C_{f(b)}(f(a))$. Also $g(a)$ is an endpoint of $S$. Hence $b a$ is not a terminal edge. (Otherwise, $f(b a)$ is terminal since $f$ is combinatorial, and so $f(a)=g(a)$, a contradiction.) Now if $g(a) \in C_{f(a)}(f(b))$, then $g(a) \in f(a) f(b)$, but $g(a)$ is an endpoint of $S$ and $f(a) f(b)$ contains no endpoints of $S$. So $g(a) \notin C_{f(a)}(f(b))$. Hence by iii), $g(a) \in C_{f(a)}\left(f\left(s_{i}\right)\right)$ for some $i=2, \cdots, n$. But $C_{a}\left(s_{i}\right)$ is a proper subset of $C_{b}(a)=C_{b}(r)$, contradicting the minimality of $C_{b}(r)$. So Case 1 cannot occur.

Case 2. ba contains a folded subedge.

By i), $b r$ and $s_{1} a$ are half-folded subedges and so there is at least one point of $F$ between $r$ and $s_{1}$ in $b a$. Label all these points $x_{1}, \cdots, x_{m}$ in order on $b a$ so that $b<r<x_{1} \leq x_{m}<s_{1}<a$ with $b x_{1}$ and $x_{m} a$ subedges, and for $i=1 \cdots(m-1)$, $x_{i} x_{i+1} \in \mathcal{E}$.

Now $g(b) \in C_{f(b)}(f(r))$, so by $1.2, g(r) \in C_{f(b)}(f(r))$. Also, for $i>1, C_{a}\left(s_{i}\right)$ is a proper subset of $C_{b}(r)$, so by the minimality of $C_{b}(r)$, we know $g(a) \notin C_{f(a)}\left(f\left(s_{i}\right)\right)$ for $i>1$. Hence $g(a) \in C_{f(a)}\left(f\left(s_{1}\right)\right)$. It follows from 1.2 that

$$
g\left(s_{1}\right) \in C_{f(a)}\left(f\left(s_{1}\right)\right) .
$$

Now since $g(r) \in C_{f(b)}(f(r))$ and $f\left(x_{1}\right)=f(b)$, we have by 1.2 that $g\left(x_{1}\right) \in$ $C_{f\left(x_{1}\right)}(f(r))=C_{f(b)}(f(r))$. But $C_{f\left(x_{1}\right)}(f(r)) \subset C_{f\left(s_{1}\right)}\left(f\left(x_{1}\right)\right)$, so

$$
g\left(x_{1}\right) \in C_{f\left(s_{1}\right)}\left(f\left(x_{1}\right)\right) .
$$

If $m=1$ then $f(a)=f\left(x_{1}\right)$. From $(*)$ above, we have $g\left(s_{1}\right) \in C_{f(a)}\left(f\left(s_{1}\right)\right)=$ $C_{f\left(x_{1}\right)}\left(f\left(s_{1}\right)\right)$. This and $(* *)$ above contradict 1.2 .

If $m>1$, then $C_{f\left(x_{1}\right)}(f(r)) \subset C_{f\left(x_{2}\right)}\left(f\left(x_{1}\right)\right)$, and so $g\left(x_{1}\right) \in C_{f\left(x_{2}\right)}\left(f\left(x_{1}\right)\right)$ and it follows from 1.2 that $g\left(x_{2}\right) \in C_{f\left(x_{2}\right)}\left(f\left(x_{1}\right)\right)$. We can repeat this argument until we get that $g\left(x_{m}\right) \in C_{f\left(x_{m}\right)}\left(f\left(x_{m-1}\right)\right)$. But $C_{f\left(x_{m}\right)}\left(f\left(x_{m-1}\right)\right) \subset C_{f\left(s_{1}\right)}\left(f\left(x_{m}\right)\right)$ so $g\left(x_{m}\right) \in C_{f\left(s_{1}\right)}\left(f\left(x_{m}\right)\right)$. Also $g\left(s_{1}\right) \in C_{f(a)}\left(f\left(s_{1}\right)\right)=C_{f\left(x_{m}\right)}\left(f\left(s_{1}\right)\right)$. But now $g$ stretches $x_{m} s_{1}$ over its $f$-image, a contradiction of 1.2 .

So $f$ is universal.

Theorem 6.2. The three properties given above in 6.1 which a combinatorial map must have in order to be universal are independent of each other.

Proof. To see this, we note first that the non-universal but combinatorial map given in Figure 3 satisfies properties ii) and iii) but not i). An edge-subedge diagram for a non-universal map $f$ satisfying i) and iii) but not ii) is given in Figure 5 . Included in the diagram are instructions (in parentheses) for constructing a map $g$ which misses $f$. (See the text near Figure 3 for details on constructing $g$.)

Finally, a map $f$ satisfying i) and ii) but not iii) is described in Figure 6.

Combining the results of sections 3,4 , and 5 , we have the following characterization of universal maps on trees.

Theorem 6.3. Let $f: R \rightarrow S$ be a map of trees, with monotone light factorization $f=l m$. Then $f$ is universal if and only if there is a subtree $R^{\prime}$ of $R$ such that $\left.{ }^{l}\right|_{m\left(R^{\prime}\right)}: m\left(R^{\prime}\right) \rightarrow S$ is a combinatorial map satisfying the following:

(i) The folded subedges of $m\left(R^{\prime}\right)$ are paired.

(ii) Each edge of $m\left(R^{\prime}\right)$ which is not a subedge contains a folded subedge. 


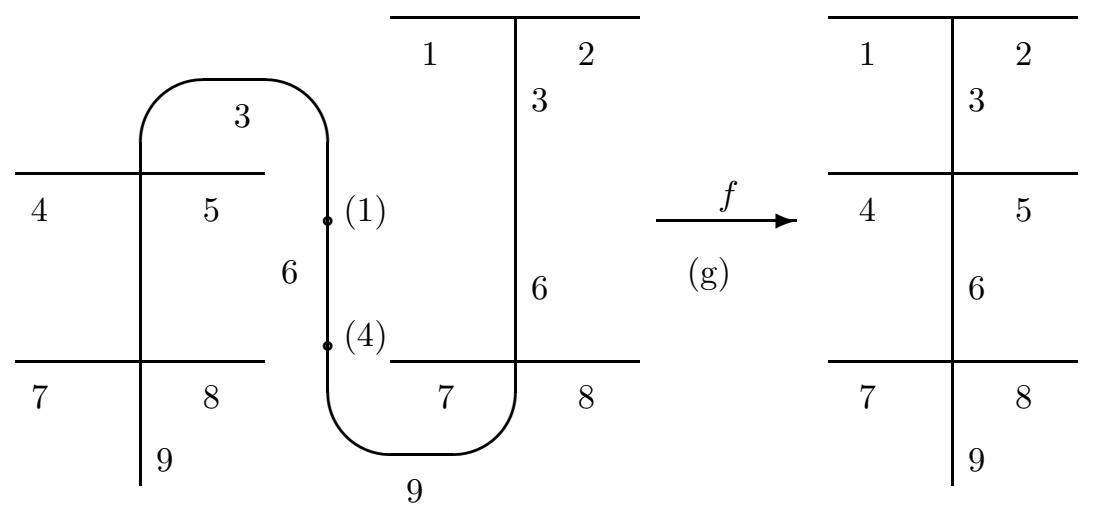

Figure 5. $f$ satisfies all but property ii).

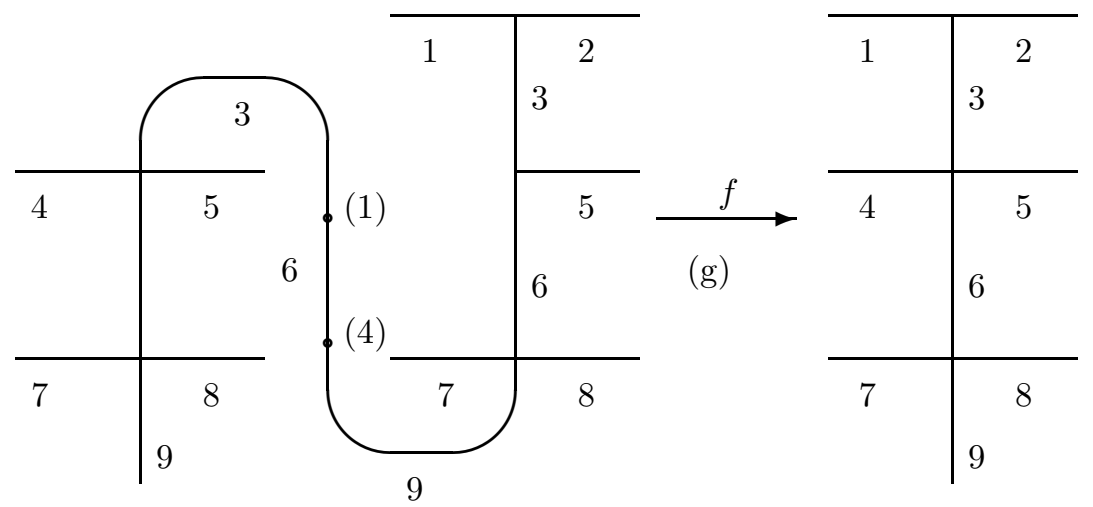

Figure $6 . f$ satisfies all but property iii).

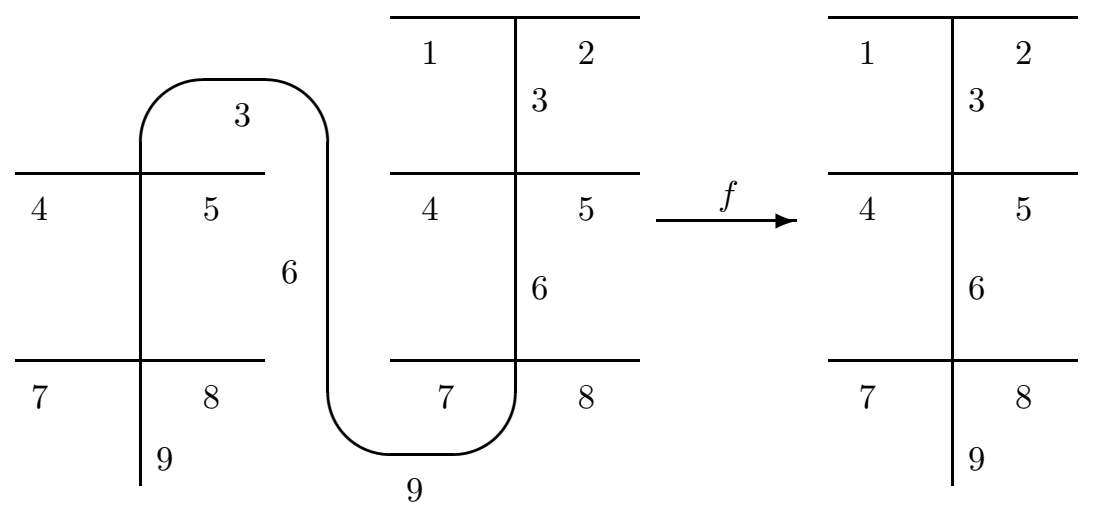

FiguRE 7. $f$ is universal but is not a $u$-map.

(iii) If $p$ is a branchpoint of order $n$ in $m\left(R^{\prime}\right)$, then $l(p)$ has order $n$ in $S$.

To conclude, we show that the class of universal maps on trees is larger than the class of $u$-maps defined by Marsh in [6]. Briefly, a $u$-map is a map of trees with five 
properties, one of which is the requirement that if $b$ is a branchpoint, then $f$ takes the star of $b$ (i.e. the union of the edges containing $b$ ) into the star of $f(b)$. Marsh shows that $\mathrm{u}$-maps are universal. The example given in Figure 7 is a universal map that is not a u-map, because it does not satisfy the above mentioned property.

\section{REFERENCES}

1. C. A. Eberhart, J. B. Fugate, and G. R. Gordh, Branchpoint Covering Theorems for Confluent and Weakly Confluent Maps, Proc. A.M.S, 55 (1976), 409-415. MR 53:14450

2. W. Holsztynski, Universal Mappings and Fixed Point Theorems, Bull. Acad. Polon. Sci., XV (1967), 433-438. MR 36:4545

3. W. Holsztynski, Universality of the product mappings onto products of $I^{n}$ and snake-like spaces, Fund. Math. 64 (1969), 147-155. MR 39:6249

4. O.W. Lokuciewski, On a theorem on fixed points, Uspehi Mat. Nauk XII 3(75) 1957, 171-172.

5. M. M. Marsh, Fixed Point Theorems for Certain Tree-like Continua, Dissertation, University of Houston, (1981).

6. M. M. Marsh, u-mappings on trees, Pacific Journal of Mathematics, Vol. 127, No. 2 (1987), 373-387. MR 88f:54067

7. S. B. Nadler, Jr., Universal Mappings and Weakly Confluent Mappings, Fund. Math, 110, (1980), 221-235. MR 82h:54057

8. G. T. Whyburn, Analytic Topology, A.M.S. Colloq. Pub. XXVIII, (1963). MR 32:425

Department of Mathematics, University of Kentucky, Lexington, Kentucky 40506

E-mail address: carl@ms.uky.edu

E-mail address: fugate@ms.uky.edu 